\title{
Optimal Portfolio Structuring in Emerging Stock Markets Using Robust Statistics
}

Fernando R. Q. Reyna**

Antonio M. Duarte Júnior ${ }^{* * *}$

Beatriz V. M. Mendes ${ }^{* * * *}$

Oscar Porto ${ }^{* * * *}$

\begin{abstract}
Emerging markets are known to have unique characteristics when compared to more developed markets. The direct use of standard mathematical models proposed and tested in more developed markets is not always recommended in emerging markets. Extreme events in emerging markets have already been verified to distort the results obtained when using standard mathematical models in several situations, including optimal portfolio structuring. Practitioners working in the asset management industry in emerging markets have not yet incorporated optimization models into their routine. One of the reasons for that is that extreme events and/or economic discontinuities (such as the Brazilian and the Argentinean devaluation crises etc.) modify the financial environment in such a way that past data become of little use when looking forward. In this article we concentrate on proposing a methodology to handle extreme events. Two numerical examples taken from the Brazilian stock market are used to illustrate the use of our proposal.
\end{abstract}

Keywords: Asset Management, Robust Portfolio, Robust Statistic, Robust Estimators.

JEL Codes: C44, C61.

\footnotetext{
${ }^{*}$ Submitted in July 2004. Revised in May 2005. Partially supported by CNPq.

${ }^{* *}$ NetQuant Tecnologia de Investimentos. E-mail: fernando@netquant.com.br

*** Diretor, Ibmec - Rio de Janeiro. E-mail: aduarte@ibmecrj.br

**** Instituto de Matemática, UFRJ. E-mail: beatriz@im.ufrj.br

***** Departamento de Engenharia Elétrica, PUC-Rio. E-mail: oporto@ele.puc-rio.br
} 


\section{Introduction}

Standard models for optimal portfolio structuring have been criticized because of their very restrictive modeling hypotheses (Chopra (1993), Gardner and Wuilloud (1995) and Michaud (1989) among others). Following that, several modeling improvements have been proposed, including the use of scenario analysis (Nemhauser et al., 1989), network structures (Crum et al., 1979), more general utility functions (Kallberg and Ziemba, 1983), the incorporation of investor's expectations (Black and Litterman, 1991), (Koskosidis and Duarte, 1997), asymmetric risk measures (Markowitz, 1959), (Harlow, 1993), large-scale models (Carino et al., 1994), (Hiller and Eckstein, 1993), etc.

Emerging markets are known to have unique characteristics when compared to more developed markets. The direct use of the standard mathematical models proposed and tested in more developed markets is not always recommended in emerging markets. For example, extreme events Embrechts et al. (1997) in emerging markets have already been verified to completely distort the results obtained when using standard mathematical models (that is, those used by practitioners in more developed markets) in several situations, such as hedging using futures contracts (Duarte and Mendes, 1998), systematic risk estimation (Duarte and Mendes, 1997), Value-at-risk computation (Duarte, 1997), volatility estimation (Mendes and Duarte, 1999), currency overlay (Duarte and Rajagopal, 1999), estimating term structures of interest rates (Almeida et al., 1998), and optimal portfolio structuring (Reyna et al., 1999b).

Practitioners working in the asset management industry in emerging markets have not yet incorporated optimization models into their routine. One of the reasons is that extreme events and/or economic discontinuities (such as the Real Economic Plan, the Mexican Crisis, the Brazilian and the Argentinean devaluation crises etc.) modify the financial environment in such a way that past data become of little use when looking forward. Portfolio optimization models must allow these changes to be easily incorporated into the methodology or, otherwise, they will remain outside the routine of local practitioners. For instance, see Reyna et al. (1999a) for a methodology to incorporate macroeconomic discontinuities in a portfolio optimization methodology with an application to international diversification in Latin American emerging markets.

In this article we propose a methodology to handle extreme events based on the theory of robust statistics (Huber, 1981). To that end, in Section 2, we review some basic concepts from robust statistical theory, prove the main results needed throughout the article, and verify that the two portfolio optimization models most widely used by practitioners in international financial markets do not provide robust optimized portfolios. Section 3 describes portfolio optimization models that can provide robust optimized portfolios, addressing an important practical question: the computational implementation of these proposals. Section 4 provides two numerical examples from the Brazilian stock markets. Our conclusions are 
presented in Section 5.

\section{Robust Statistics Applied to Portfolio Optimization}

Definition 1 The general asset management model (GAMM) is defined in this work as

$$
\begin{aligned}
\operatorname{Maximize} & U(w) \\
\text { subject to : } \quad & =R x \\
e^{T} x & =c_{0} \\
x & \in \mathcal{R}_{+}^{n} \\
w & \in \mathcal{R}^{m}
\end{aligned}
$$

where $n$ is the number of assets; $m$ is the number of scenarios; $U(\cdot)$ is a utility function; $x$ represents the composition of the portfolio; $w$ is the wealth obtained under each scenario; $c_{0}$ is the initial wealth available for investing; $R=R^{*}+E$, where $R^{*}$ is a $m \times n$ matrix of returns for all $m$ scenarios and each of the $n$ assets under consideration, and $E$ is a matrix of 1 's. For instance, $\left(R^{*}\right)_{i j}$ is the expected return for asset " $j$ " under the hypothesis of scenario " $i$ ", and $e \in \mathcal{R}^{n}$ is a vector of 1 's.

Definition 2 If $x^{*}$ is an optimal solution for the GAMM, it represents an optimal portfolio composition.

Definition 3 A statistical utility function (SUF) is any utility function which can be put as a linear combination of statistical estimators. A SUF can be written as

$$
U(\cdot)=\sum_{k=1}^{l} \beta_{k} T_{k}(\cdot)
$$

where $\beta_{k} \in \mathcal{R}$ and $T_{k}(\cdot)$ is a statistical estimator.

Definition 4 Given a sample $y=\left(y_{1}, y_{2}, \ldots, y_{m}\right)$, we define a contaminated sample $y^{\prime}=\left(y_{1}^{\prime}, y_{2}^{\prime}, \ldots, y_{m}^{\prime}\right)$ as a sample obtained by replacing any set of $p$ (with $p<m$ ) observations in by arbitrary values.

Definition 5 We define the maximum bias that can be caused by a contaminated sample on an estimator $T(\cdot)$ as

$$
b(p, T, y)=\sup _{\underline{y^{\prime}}}\left\|T\left(\underline{y^{\prime}}\right)-T(\underline{y})\right\|
$$


where $\|\cdot\|$ is a norm, the "sup" is taken over all contaminated samples, and $p$ is the number of contaminating values.

Definition 6 The finite sample replacement breakdown point is defined as

$$
\varepsilon_{m}^{*}(T, \underline{y})=\min _{\frac{p}{m}}\left\{b\left(p, T, \underline{y^{\prime}}\right)=\infty\right\}
$$

where $m$ is the sample size and $p$ is the number of contaminating values.

The finite sample replacement breakdown point definition was given in Donoho and Huber (1983). They define the breakdown point as the minimum proportion of observations of a sample that, if replaced by arbitrary values, can make the maximum bias of $T(\cdot)$ tend to infinity, or $T(\cdot)$ not to exist.

Definition 7 Given $T(\cdot)$ and $y^{\prime}$, we define as the least favorable sample (LFS) any sample $y$ for which $b(p, T, y)=\infty$ for a given $p$.

Theorem 1 The breakdown point of a SUF equals the smallest breakdown point among all estimators composing the SUF.

Proof See appendix.

Definition 8 Given a random variable $Y$, with distribution $F_{Y}$, we define a contaminating process for $Y$ as the process

$$
Y(\varepsilon, H) \sim(1-\varepsilon) F_{Y}+\varepsilon F_{H}
$$

where $F_{H}$ is the distribution of an arbitrary random variable $H$, and $\varepsilon \in(0,1)$.

For the purposes of this work one can consider the existence of an atypical observation for a financial time series as a contaminating process. That is, the existence of an atypical observation for the return (gain or loss) of an asset can be regarded as the process of replacing a typical observation by an arbitrary value.

At this point it is important to observe that the contaminating process is related to the elements of the scenario matrix $R^{*}$, but the result proved in Theorem 1 depends on the contamination of the elements in $w$. It is straightforward to verify that a contaminating process for $w$ is generated from a contaminating process for the columns of the scenario matrix $R^{*}$ for any feasible solution of model (1).

As a consequence, when using a GAMM for structuring an optimal portfolio, if the SUF used is not robust, and if the scenarios used for the returns of the assets under consideration present outliers, the resulting optimal portfolio composition may be distorted by atypical observations. 
Definition 9 A real portfolio is a portfolio obtained from a GAMM with a noncontaminated sample.

Definition 10 The robust optimal portfolio is a portfolio obtained from a GAMM with a SUF with breakdown point greater than the contamination proportion existing in $w$.

Based on the theoretical structure defined above, we can prove that the optimized portfolio obtained from the mean-variance model (Markowitz (1959)) is not a robust portfolio. The mean-variance model is obtained by substituting in (1)

$$
U(w)=\frac{1}{m} \sum_{i=1}^{m} w_{i}-\frac{\lambda}{m} \sum_{i=1}^{m}\left(w_{i}-\frac{\sum_{i=1}^{m} w_{i}}{m}\right)^{2}
$$

where all scenarios are assumed to have equal probability $\left(=\frac{1}{m}\right), w_{i}$ is the wealth under scenario $i$, and $\lambda$ is the investor's risk aversion parameter.

Corollary 1 The optimal portfolio $x^{*}$ obtained from the mean-variance model is not a robust portfolio.

Proof See appendix.

The GAMM is being increasingly used by practitioners in international financial markets. Usually, the difference between the models is related only to the SUF chosen. One of the most used SUF is

$$
U(w)=\frac{1}{m} \sum_{i=1}^{m} w_{i}-\frac{\lambda}{m} \sum_{i=1}^{m} \max \left\{0 ; c_{0}(1+\mathrm{MAR})-w_{i}\right\}
$$

where MAR is the investor's minimal acceptable return in a downside risk portfolio optimization framework, as proposed by Harlow (1993), and all other parameters/variables have already been defined. The mean-downside risk model is obtained by substituting (7) in (1).

Corollary 2 The optimal portfolio $x^{*}$ obtained from the mean-downside risk model is not a robust portfolio.

Proof See appendix.

That is, the two portfolio optimization models most widely used by practitioners in international financial markets - the mean-variance model and the meandownside risk model - do not provide robust optimal portfolios. We need to search 
for a framework that allows us to "generate" portfolio optimization models which can provide robust optimal portfolios.

\section{The GAMM and its Robust Version}

Although we do not intend to present the most comprehensive framework for portfolio optimization models that can provide robust optimal portfolios in this article, Theorem 3 helps us in this task: in order to propose a GAMM which can provide robust optimal portfolios we need to modify the SUF, using, to that end, only statistical estimators that possess high breakdown points (for instance, $50 \%$ ).

For instance, one can use a SUF such as

$$
U(w)=\operatorname{median}_{1 \leq i \leq m}\left\{w_{i}\right\}-\lambda \operatorname{median}_{1 \leq i \leq m}\left\{\max \left\{0 ; c_{0}(1+\mathrm{MAR})-w_{i}\right\}\right\}
$$

We define a GAMM with a SUF such as (8) as the median-median downside risk (MMDR) model. This is an interesting model, as verified next.

Corollary 3 The optimal portfolio $x^{*}$ obtained from the MMDR model is a robust portfolio.

Proof See appendix.

\subsection{Computational implementation}

A difficult problem in robust statistics estimation, and in particular in robust regression, is that of computing estimators based on minimization of the $k^{t h}$ smallest value of absolute residuals, because of their combinatorial complexity (Bocvek and Lachout, 1995). It can be verified that the MMDR model is a specific case of this kind of problem (Reyna, 2001).

The MMDR can be formulated in a mixed integer programming framework as

$$
\begin{aligned}
& \text { Maximize } w_{\text {median }}-\lambda d_{\text {median }} \\
& \text { subject to: } w_{\text {median }}<w_{i}+M s_{i} \quad \forall i=1,2, \cdots, m \\
& w_{\text {median }}>w_{i}-M+M s_{i} \forall i=1,2, \cdots, m \\
& d_{\text {median }}<d_{i}^{-}+M z_{i} \forall i=1,2, \cdots, m \\
& d_{\text {median }}>d_{i}^{+}-M+M z_{i} \forall i=1,2, \cdots, m \\
& d^{-}+w=d^{+}+c_{0}(1+\mathrm{MAR}) e \\
& e^{T} s<m \\
& e^{T} z>m+1 \\
& w=R x \\
& e^{T} x=c_{0} \\
& x_{j} \geq 0 \forall j=1,2, \cdots, n \\
& w_{i}, d_{i}^{+}, d_{i}^{-} \geq 0 \forall i=1,2, \cdots, m \\
& s_{i}, z_{i} \in\{0 ; 1\} \forall i=1,2, \cdots, m
\end{aligned}
$$




$$
w_{\text {median }}, d_{\text {median }} \in \mathcal{R}
$$

where $w_{\text {median }}$ represents the median wealth obtained under the hypotheses of each scenario, $d_{\text {median }}$ represents the median downside risk measure, $s, z, d^{+}$are all auxiliary variables, $M$ is a "big constant" chosen in accordance to the "Big $M$ " method (Luenberger, 1984), and all other parameters/variables have been defined before.

The idea behind the formulation in (9) is that the process of calculating the median of a sample is equivalent to sorting out the sample and getting the value that is in the middle of the sorted sample. This means that we will have fifty percent of the sample below and fifty percent above the median: that is why we need the binary variables $s$ and $z$, in order to "control" the proportion of the sample below and above $w_{\text {median }}$ and $d_{\text {median }}$.

From the optimization point of view, model (9) is a difficult problem to solve, because of its computational complexity. This complexity is directly related to the number of scenarios $(m)$ and assets $(n)$ used in the optimization process: the larger these values, the more difficult it becomes to obtain the robust optimized portfolio. For implementational details please refer to Reyna (2001).

\section{Numerical Examples}

In this section we provide two numerical examples:

- The first one is based on the hypothesis that we know the true probability distribution function of all assets' returns, and we use a contamination process for this distribution.

- The second one uses historical data, with a specific atypical event. The objective is to "simulate" the real world and to illustrate the usefulness of our proposal in practice.

In both numerical experiments we use daily data from the Brazilian equity market from December 1st, 1998, to May 7th, 1999. This period was chosen because it covers the Brazilian currency devaluation crisis of January, 1999. The assets considered for the analyses were the ten most traded stocks in the São Paulo Stock Exchange during that period of time (presented below in alphabetical order):

- Bradesco PN;

- Banco do Brasil PN;

- Cemig PN;

- Eletrobrás ON;

- Eletrobrás PNB;

- Petrobrás PN; 
- Telebrás ON;

- Telebrás PN;

- Telesp PN;

- CVRD PNA.

The riskless asset is represented by local short-term interest rates.

\subsection{The first numerical example}

The main assumption in this first example is that we know the probability distribution of all assets considered for investing: a multivariate normal distribution. This family of distributions needs only two parameters for its full characterization: the mean vector $(\mu)$ and the covariance matrix $\left(\sum\right)$. For estimating these parameters we use all historical data available. We also rely on a robust estimation technique: the minimum covariance determinant (Rousseeuw and Zomeren, 1990). This procedure provides the robust estimators for $\mu$ and $\sum$.

\subsubsection{Scenario generation}

With the estimates just obtained we generate a two-month sample (that is, forty-two business days) of returns for all assets under consideration using a Monte Carlo method. We generate one hundred sets of two-month samples with the same probability, from now on referred to as "clean scenarios". At the end of the scenario generation process we end up with one hundred "clean scenarios".

\subsubsection{Contaminating process}

The next step consists of contaminating the "clean scenarios" using outliers. To that end, our choice was to use the outliers found in the historical data. These outliers can be identified using a robust distance concept (Rousseeuw, 1984). According to this distance these historical outliers are ordered and introduced in the "clean scenarios" according to the degree of contamination desired. In the numerical results presented below the samples contained $5 \%, 25 \%, 40 \%$ or $50 \%$ of contamination. After contamination the "clean scenarios" become the "contaminated scenarios".

\subsubsection{Models}

We compare two portfolio optimization models:

- The first one is the mean-downside risk model, mentioned before. We shall refer to this model as the "classical model". 
- The second one is the MMDR model, presented above. We shall refer to this model as the "robust model".

In both cases we set $\lambda=1$.

\subsubsection{Steps}

The two steps of this first numerical experiment are:

- To run both models for the 100 contaminated samples for all different levels of contamination (that is, those with $5 \%, 25 \%, 40 \%$ and $50 \%$ of outliers).

- To compare the portfolios' optimal compositions obtained for different samples, assessing the effect of the contamination proportion on the two models.

\subsubsection{Results}

Figure 1 and Figure 2 compare the portfolio allocation in one of the stocks considered (CEMIG) for each of the one hundred 5\% "contaminated scenarios", when using the two models mentioned above. Figure 3 and Figure 4 provide the similar results for each of the one hundred $25 \%$ "contaminated scenarios".

Clearly, while in the case of the "classical model" the optimal allocation varies arbitrarily between $0 \%$ and $100 \%$ for the different scenarios used, in the case of the "robust portfolio" it remains fairly stable for the two levels of contamination depicted. Also notice that the allocation provided by the "robust model" varies slightly more in the case of the $25 \%$ "contaminated scenarios" when compared to the $5 \%$ "contaminated scenarios".

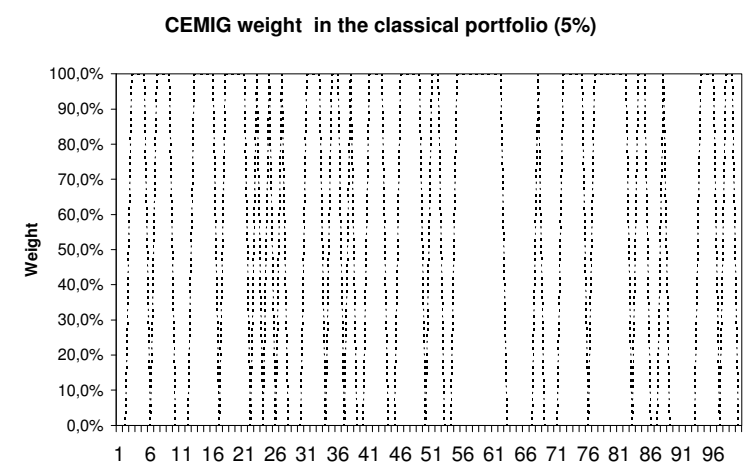

Figure 1

CEMIG's optimal allocation using the "classical model" and the $5 \%$ "contaminated scenarios" 
CEMIG weight in the robust portfolio (5\%)

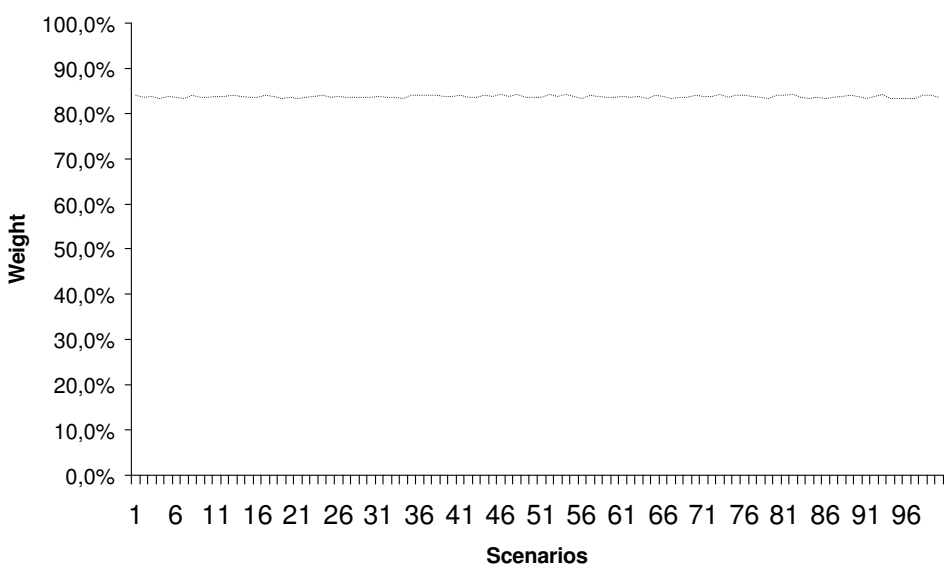

Figure 2

CEMIG's optimal allocation using the "robust model" and the $5 \%$ "contaminated scenarios"

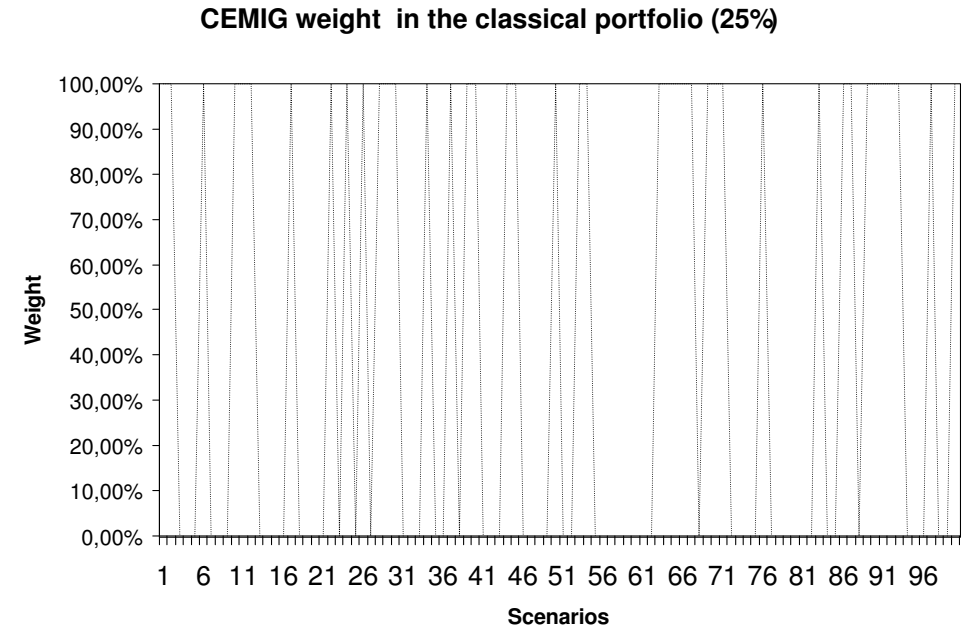

Figure 3

CEMIG's optimal allocation using the "classical model" and the $25 \%$ "contaminated scenarios" 


\section{CEMIG weight in the robust portfolio (25\%)}

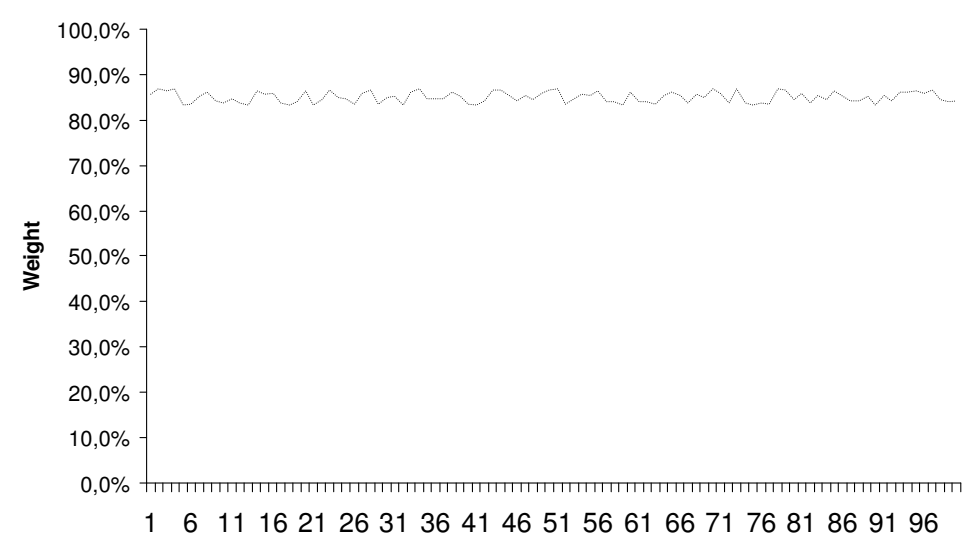

Figure 4

CEMIG's optimal allocation using the "robust model" and the $25 \%$ "contaminated scenarios"

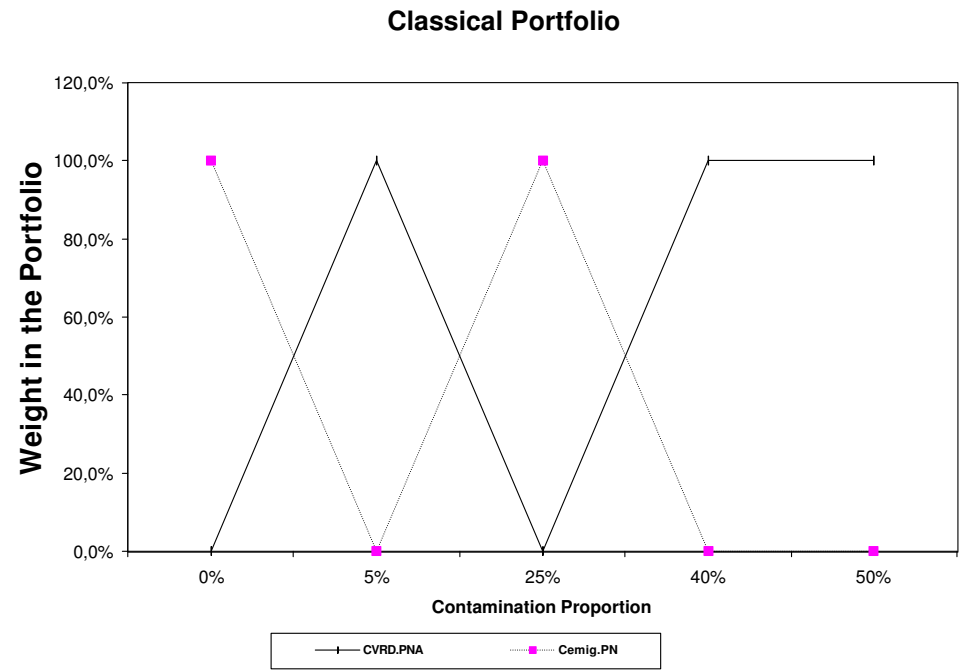

Figure 5

Optimal allocation for two stocks using the "classical model" and an increasing contamination proportion 


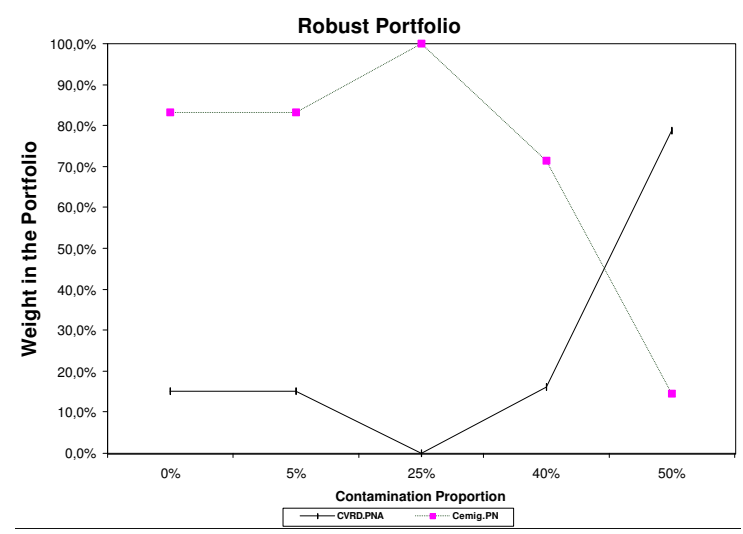

Figure 6

Optimal allocation for two stocks using the "robust model" and an increasing contamination proportion

We now observe the effect of higher contamination proportions. For a randomly chosen sample, Figure 5 shows, for the "classical model", the behavior of the optimal allocation for two stocks (CEMIG and CVRD) as the percentage of contamination increases (from $0 \%$, to $5 \%$, to $25 \%$, to $40 \%$, and finally to $50 \%$ ). Figure 6 shows the corresponding result obtained when using the "robust model", instead of the "classical model".

We can note a more stable pattern of the optimal weights obtained from the "robust model" when compared to the "classical model" as the contamination proportion increases to the value of the breakdown point (50\%).

The six figures exhibited so far clearly show that the optimal allocation obtained from the "classical model" varies much more than those obtained from the "robust portfolio" as the contamination proportion increases. As a consequence, we can expect the optimal allocation proposed by a "robust model" to remain more stable during/following extreme events. Remember that this was exactly our objective when proposing this new approach to optimal portfolio structuring.

\subsection{The second numerical experiment}

Using the data available, in this experiment a window of 42 days is used to define the historical scenarios. This window is shifted every day so that, at each step, a new sample is obtained by the incorporation of a new observation, and by the withdrawal of the oldest one. A total of 40 samples were generated.

In our case, the initial sample contains the days corresponding to the Brazilian currency devaluation. The objective of this numerical example is to understand the practical effect of atypical events on the optimal allocations proposed by the 
two models.

For all 40 windows, we optimize both models. At each step, the next-day returns (thus, out of the sample) are used to obtain the marked-to-the-market returns for the optimal portfolios proposed by the "robust model" and by the "classical model". By moving the window, and following the portfolios' recommendations for optimal allocation, a set of 40 out-of-sample portfolio returns were obtained.

\subsubsection{Results}

Figure 7 depicts the out-of-sample returns obtained for the two models. The first comment is that the returns posted by the two models are not much different, except for the period around the end of March, 1999: that is the period when the effects of the Brazilian devaluation start leaving the historical scenarios used for the optimizations. That is, around the end of March, 1999, almost two months after the devaluation, the optimal allocations provided by the "robust model" tend to change smoothly, this not being the case for the "classical model". As a consequence, since the stock market returns during that period remained fairly stable, the returns posted by the "robust model" change smoothly too, this not being the case, again, for the "classical model". These comments corroborate the results obtained in the first numerical example.

Finally, in Table 1, we observe that the mean and the accumulated out-ofsample returns obtained with the "robust model" were higher than the ones obtained with the "classical model", for basically the same level of out-of-sample volatility. These results illustrate a better ex-post performance for the "robust model" when compared to the "classical model".

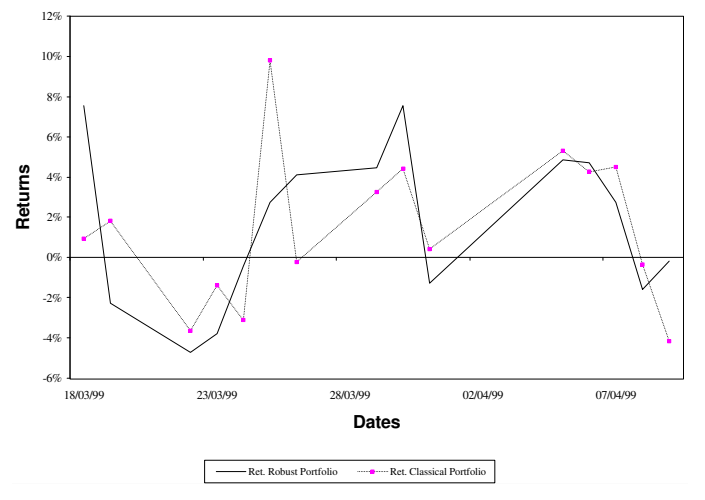

Figure 7

Out-of-sample returns for two models 
Table 1

Statistics for out-of-sample returns of two models

\begin{tabular}{lcc}
\hline & "Classical model" & "Robust model" \\
\hline Mean return & $1.453 \%$ & $1.633 \%$ \\
Accumulated return & $22.936 \%$ & $26.167 \%$ \\
Standard deviation & $0.038 \%$ & $0.039 \%$ \\
\hline
\end{tabular}

\section{Conclusion}

Asset managers need to make strategic and tactical investment decisions: the strategic decisions are related to the process of elaborating a long term investment policy; the tactical decisions are related to the process of elaborating short term decisions. Although the proposal presented in this article can handle both types of decisions, it is more suited to strategic decisions.

The strategic asset allocation is more concerned with the "essence" of assets return-risk behavior, instead of atypical events that the financial markets may occasionally present, distorting the short-term performance of assets. Since robust procedures are typically concerned with the pattern followed by the majority of the data, this characteristic is one of the main incentives for using the proposed robust optimal portfolios for strategic asset allocation. For instance, it is not reasonable for institutional investors to alter all their investment policy (say, devised for an investment horizon of decades) just because of a few atypical observations.

The optimization computations required by our proposal present a higher level of computational difficulty when compared to the computations required by the portfolio structuring methodologies used by practitioners nowadays. More research on more efficient computational solutions for large-scale mixed integer programming models is needed. Until new developments are made for large-scale mixed integer programming, our proposal will remain useful only when applied to small portfolio structuring models, as in our numerical examples.

Finally, the concepts developed in this article can be easily adapted to other applications out of financial markets, such as robust crew scheduling, robust production planning, and robust assignment, to mention only three possibilities.

\section{References}

Almeida, C. I. R., Duarte, Júnior, A. M., \& Fernandes, C. A. C. (1998). Decomposing and simulating the movements of term structures of interest rates in emerging Eurobond markets. Journal of Fixed Income, 8:21-31.

Black, F. \& Litterman, R. (1991). Asset allocation: Combining investors' views with market equilibrium. Journal of Fixed Income, 1:21-34.

Bocvek, P. \& Lachout, P. (1995). Linear programming approach to LMS estimation. Computational Statistics \& Data Analysis, 19:129-134. 
Carino, D., Kent, T., Myers, D., Stacy, C., Sylvanus, M., Turner, A., Watanabe, K., \& Ziemba, W. (1994). The Russel-Yasuda Kasai model: An asset-liability model for a Japanese insurance company using multistage stochastic programming. Interfaces, 24:29-49.

Chopra, V. K. (1993). Improving optimization. Journal of Investing, 2:51-59.

Crum, D. R., Kligman, D. D., \& Tavis, L. A. (1979). Implementation of largescale financial planning models: Solution efficient transformations. Journal of Financial and Quantitative Analysis, 13:87-102.

Donoho, D. \& Huber, P. (1983). The notion of breakdown point. In A Festschrift for Erich Lehmann. Wadsworth, Belmont.

Duarte, Júnior, A. M. (1997). Model risk and risk management. Derivatives Quarterly, 3:60-72.

Duarte, Júnior, A. M. (1999). Fast computation of efficient portfolios. Journal of Risk, 1:71-94.

Duarte, Júnior, A. M. \& Mendes, B. V. M. (1997). Robust estimation of systematic risk in emerging stock markets. Emerging Markets Quarterly, 1:83-95.

Duarte, Júnior, A. M. \& Mendes, B. V. M. (1998). Robust hedging using futures contracts with an application to emerging markets. Journal of Derivatives, $6: 75-95$

Duarte, Júnior, A. M. \& Rajagopal, R. (1999). A scenario-based approach to optimal currency overlay. Journal of Portfolio Management, 25:51-59.

Embrechts, P., Kluppelberg, C., \& Mikosch, T. (1997). Modelling Extremal Events. Springer-Verlag, Berlin.

Gardner, G. \& Wuilloud, T. (1995). Currency risk in international portfolios: How satisfying is optimal hedging. Journal of Portfolio Management, 21:59-67.

Harlow, W. V. (1993). Asset allocation in a downside risk framework. Financial Analysts Journal, 49:14-26.

Hiller, R. \& Eckstein, J. (1993). Stochastic dedication: Designing fixed-income portfolios using massively parallel benders decomposition. Management Science, $39: 1422-1438$

Huber, P. J. (1981). Robust Statistics. John Wiley, New York.

Kallberg, J. G. \& Ziemba, W. T. (1983). Comparison of alternative utility functions in portfolio selection problems. Management Science, 29:1257-1276. 
Koskosidis, Y. A. \& Duarte, Jr., A. M. (1997). A scenario-based approach to active asset allocation. Journal of Portfolio Management, 23:74-85.

Luenberger, D. (1984). Linear and Nonlinear Programming. Addison Wesley, Reading.

Markowitz, H. M. (1959). Portfolio Selection: Efficient Diversification of Investments. John Wiley, New York.

Mendes, B. V. M. \& Duarte, Jr., A. M. (1999). Robust estimation for ARCH models. Brazilian Review of Econometrics, 19:139-180.

Michaud, R. (1989). The Markowitz optimization enigma: Is optimized optimal? Financial Analysts Journal, 45:31-42.

Nemhauser, G. L., Rinnooy, A. H. G., \& Todd, M. J. (1989). Operations Research and Management Science: Optimization. North Holland, Amsterdam.

Reyna, F. R. Q. (2001). Carteira de Investimento Robusta. PhD thesis, Pontifícia Universidade Católica, Rio de Janeiro.

Reyna, F. R. Q., Duarte, Júnior, A. M., \& Migon, H. S. (1999a). International diversification with incomplete data. Journal of Financial Engineering, 8:9-30.

Reyna, F. R. Q., Mendes, B. V. M., \& Duarte, Júnior, A. M. (1999b). Estruturación de carteras de inversiones con una aplicación en los mercados emergentes Latino-Americanos de acciones. Estadística, 49:167-186.

Rousseeuw, P. (1984). Least median of squares regression. Journal of American Statistical Association, 79:871-880.

Rousseeuw, P. \& Zomeren, B. C. (1990). Unmasking multivariate outliers and leverage points. Journal of American Statistical Association, 85:633-639. 


\section{Appendix}

\section{Proof of Theorem 1}

Let $\frac{p^{*}}{m}$ denote the breakdown point of $U(\cdot)$. If $\frac{p_{k}}{m}$ denotes the breakdown point of the $k^{t h}$ estimator in (2), we can assume without loss of generality that

$$
\frac{p_{1}}{m} \leq \frac{p_{2}}{m} \leq \cdots \leq \frac{p_{l}}{m}
$$

Using the triangle inequality we obtain

$$
\begin{aligned}
b(p, U, w) & =\sup _{w^{\prime}}\left\|U\left(w^{\prime}\right)-U(w)\right\| \\
& =\sup _{w^{\prime}}\left\|\sum_{k=1}^{l} \beta_{k} T_{k}\left(w^{\prime}\right)-\sum_{k=1}^{l} \beta_{k} T_{k}(w)\right\| \\
& =\sup _{w^{\prime}}\left\|\sum_{k=1}^{l} \beta_{k}\left(T_{k}\left(w^{\prime}\right)-T_{k}(w)\right)\right\| \\
& \leq \sum_{k=1}^{l}\left|\beta_{k}\right| \sup _{w^{\prime}}\left\|T_{k}\left(w^{\prime}\right)-T_{k}(w)\right\| \\
& =\sum_{k=1}^{l}\left|\beta_{k}\right| b\left(p, T_{k}, w\right)
\end{aligned}
$$

Assuming that

$$
\frac{p^{*}}{m}<\frac{p_{1}}{m}
$$

we obtain from (A.2) that

$$
+\infty=b\left(p^{*}, U, w\right) \leq \sum_{k=1}^{l}\left|\beta_{k}\right| b\left(p^{*}, T_{k}, w\right)
$$

meaning that at least one $b\left(p^{*}, T_{k}, w\right)$ must equal $+\infty$. That cannot be the case, given Definition 6, (A.1) and (A.3). We have that

$$
\frac{p^{*}}{m} \geq \frac{p_{1}}{m}
$$

In order to conclude this proof we verify that

$$
\frac{p^{*}}{m} \leq \frac{p_{1}}{m}
$$

By contradiction, assume that

$$
\frac{p_{1}}{m}<\frac{p^{*}}{m}
$$

Brazilian Review of Econometrics 25(2) November 2005 
Then

$$
b\left(p_{1}, U, w\right)<+\infty
$$

If $w_{1}^{\prime}$ denotes the least favorable sample for $T_{1}(\cdot)$,

$$
\begin{aligned}
b\left(p_{1}, U, w_{1}^{\prime}\right) & =\sup _{w^{\prime}}\left\|\sum_{k=1}^{l} \beta_{k} T_{k}\left(w^{\prime}\right)-\sum_{k=1}^{l} \beta_{k} T_{k}\left(w_{1}^{\prime}\right)\right\| \\
\geq \mid-\sup _{w^{\prime}} \| \sum_{k=2}^{l} \beta_{k} T_{k}\left(w^{\prime}\right) & -\sum_{k=2}^{l} \beta_{k} T_{k}\left(w_{1}^{\prime}\right)\left\|+\left|\beta_{1}\right| \sup _{w^{\prime}}\right\| T_{1}\left(w^{\prime}\right)-T_{1}\left(w_{1}^{\prime}\right) \| \mid
\end{aligned}
$$

If $p_{1}<p_{2}$, and since

$$
\sup _{w^{\prime}}\left\|T_{1}\left(w^{\prime}\right)-T_{1}\left(w_{1}^{\prime}\right)\right\|=b\left(p_{1}, T_{1}, w_{1}^{\prime}\right)=+\infty
$$

one concludes that there is a contradiction with (A.8), implying (A.6).

If $p_{1}=p_{2}$ one has to apply (A.9) slightly modified twice (for $w_{1}^{\prime}$ and $w_{2}^{\prime}$ ) to contradict (A.8). The extension is obvious for $p_{1}=p_{3}$, and so on.

\section{Proof of Corollary 1}

As originally formulated in Markowitz (1959), the mean-variance model is

$$
\begin{gathered}
\text { Maximize } \mu^{T} x-\lambda x^{T} \sum x \\
\text { subject to: } e^{T} x=c_{0} \\
x \in \mathcal{R}_{+}^{n}
\end{gathered}
$$

where $\mu$ and $\sum$ are maximum likelihood estimators for the mean return and covariance of the returns of all $n$ assets under consideration. It can be verified (Duarte, 1999) that (A.11) is equivalent to (1) with the utility function given in (6).

Using Theorem 1, it is sufficient to verify that one of the real-valued statistical estimators in (6) has a breakdown point equal to zero. Huber (1981) verifies that

$$
T_{1}(w)=\frac{1}{m} \sum_{i=1}^{m} w_{i}
$$

has a breakdown point equal to zero.

\section{Proof of Corollary 2}

The mean-downside risk model has a SUF with (7), which has a breakdown point equal to zero (Huber, 1981). The result follows from Theorem 1. 


\section{Proof of Corollary 3}

Observe that the SUF (8) can be simplified to

$$
U(\cdot)=\operatorname{median}(\cdot)-\lambda \operatorname{median}(\cdot)
$$

Huber (1981) verifies that the median estimator has a breakdown point equal to $50 \%$. Invoking Theorem 1 we conclude that the SUF (8) has a breakdown point equal to $50 \%$, which is the maximum amount possible. 